\title{
SUFFICIENT CONDITIONS FOR ONE-SIDED OPERATORS
}

\author{
M. S. Riveros, L. De Rosa, And A. DE LA Torre
}

\begin{abstract}
In this paper we give sufficient conditions on pair of weights $(w, v)$ for some one-sided operators to be bounded from $L^{p}\left(v^{p}\right)$ to $L^{p}\left(w^{p}\right)$. The operators we deal with include the one-sided fractional maximal operator and the one-sided singular integrals. For the first operator necessary and sufficient conditions are knwon (see $[\mathrm{MT}],[\mathrm{LT}]$ ). These conditions usually amount to check the boundedness of the operator on functions that are powers of the weights and are hard to check. Our conditions are of $A_{p}$ type and are therefore easy to verify. Similar results for two sided operators were obtained by $\mathrm{C}$. Pérez in [P1] and [P2].
\end{abstract}

\section{INTRODUCTION}

In this paper we study two weight norm inequalities for one-sided operators. We consider a generalized version of one-sided fractional maximal operators, one-sided maximal operators associated to a Banach function space and the one-sided singular integrals introduced by $[\mathrm{AFM}]$.

The two weight problem in the first case, i.e. maximal functions have been solved in [S] and [MT]. In these papers conditions are given that are necessary and sufficient for the boundedness of the operators. However the solution is unsatisfactory in the sense that the conditions are hard to check. On the other hand there are remarkably simple conditions of $A_{p}$ type that give weak type of the operators. We will show that there are conditions close to $A_{p}$ that are sufficient for the strong type of the operators. These conditions are given in terms of norms associated to Banach function spaces. We refer to [P1] for definitions and main properties of these spaces. Our results can be considered as the one-sided versions of those obtained by C. Pérez in [P1], and [P2].

The paper is organized as follows: in section 2 we introduce the notation, state the results and give some applications to the one-sided singular integrals, and finally in section 3 we give the proofs of the results.

We finish this introduction with some notation. A weight $w$ will be a nonnegative locally integrable function. For any measurable set $E, w(E)$ will denote the integral of $w$ over $E$ and the letter $C$ will be a constant that may change from time to time.

1991 Mathematics Subject Classification. Primary 42B25, 26A33.

Key words and phrases. One-sided weights, Banach function spaces, singular integrals, maximal fractional integrals.

Research supported by D.G.I.C.Y.T. (PB94-1496), Junta de Andalucía, Universidad de Buenos Aires and Universidad Nacional de Córdoba. 


\section{Statement of the Results}

Let $X$ be a Banach function space over $\mathbb{R}$. If $f$ is any measurable function and $I$ is any interval we define the $X$-average of $f$ over $I$ as

$$
\|f\|_{X, I}=\left\|\delta_{|I|}\left(f \chi_{I}\right)\right\|_{X}
$$

where $\delta_{s}$ is the dilation operator $\delta_{s} f(x)=f(s x)$ defined for any $s>0$.

If $X^{\prime}$ is the associated space, then the generalized Hölder's inequality

$$
\frac{1}{|I|} \int_{I}|f g| \leq\|f\|_{X, I}|| g \|_{X^{\prime}, I}
$$

holds. We will also ask for this Banach function space, $X$, that there exists a constant $C>0$ such that for every $a<b<c$, with $c-b>b-a$, the inequality

$$
\|f\|_{X,(b, c)} \leq C\|f\|_{X,(a, c)}
$$

holds. The one-sided maximal operator associated to $X$ is defined as

$$
M_{X}^{+} f(x)=\sup _{b>x}\|f\|_{X,(x, b)} .
$$

We begin with a maximal operator that includes both the one-sided Hardy-Littlewood maximal operator and the one-sided fractional maximal operator. We will consider from now on, a function $h(x, y)$ defined on $y>x$, non-negative, continuous, non-increasing on the second variable and such that

(1) $\lim _{(y-x) \rightarrow 0}(y-x) h(x, y)=\ell$ exists and,

(2) there exists $0<\beta \leq 1$ such that $(y-x)^{\beta} h(x, y)$ is bounded.

We define the one-sided maximal operators associated to $h$ as

$$
M_{h}^{+} f(x)=\sup _{c>x} h(x, c) \int_{x}^{c}|f(t)| d t \text { and } M_{X, h}^{+} f(x)=\sup _{c>x}(c-x) h(x, c)\|f\|_{X,(x, c)} .
$$

Note that if $h(x, c)=(c-x)^{-1}$, then $M_{h}^{+} f=M^{+} f$, and $M_{X, h}^{+} f=M_{X}^{+} f$. If $h(x, c)=(c-x)^{\alpha-1}$, then $M_{h}^{+} f=M_{\alpha}^{+} f$, the one-sided fractional maximal operator studied in $[\mathrm{AnS}]$ and [MOT]. We also observe that $M_{h}^{-}$and $M_{X, h}^{-}$are defined in an analogous way and clearly every theorem has a corresponding one, reversing the orientation of $\mathbb{R}$. Our first theorem is the following.

Theorem 2.2. Let $1<p<\infty$, and let $X$ be a Banach function space, with associated space $X^{\prime}$ such that $M_{X}^{+}: L^{p}(\mathbb{R}) \rightarrow L^{p}(\mathbb{R})$ is bounded. We assume that the pair of weights $(w, v)$ satisfies the condition:

there exists $C$ such that

$$
\left(A_{p, X, h}^{+}\right) \quad\left(\frac{1}{b-a} \int_{a}^{b} w^{p}\right)^{\frac{1}{p}}\left\|(c-b) h(b, c) v^{-1}\right\|_{X^{\prime},(b, c)} \leq C
$$

for all $a<b<c: b-a<c-b$.

Then, there exists a constant $C$ such that

$$
\left(\int_{\mathbb{R}}\left[M_{h}^{+} f\right]^{p} w^{p} d x\right)^{\frac{1}{p}} \leq C\left(\int_{\mathbb{R}}|f|^{p} v^{p} d x\right)^{\frac{1}{p}}
$$

for every $f$.

In the case $h(x, c)=(c-x)^{-1}$, we will denote $A_{p, X, h}^{+}$by $A_{p, X}^{+}$. Theorem 2.2 raises the question of finding Banach function spaces, for which $M_{X}^{+}$is bounded. A partial answer is given by the following theorem. 
Theorem 2.3. Let $1<p<\infty$, and $X$ be a Banach function space. We consider the following statements.

a) $M_{X}^{+}: L^{p}(\mathbb{R}) \rightarrow L^{p}(\mathbb{R})$ is bounded.

b) There is a constant $C$ such that for all $h$ as above,

$$
\int_{-\infty}^{\infty}\left[M_{h}^{+} f\right]^{p} \frac{1}{\left[M_{X^{\prime}, h}^{+}\left(u^{1 / p}\right)\right]^{p}} \leq C \int_{-\infty}^{\infty}|f|^{p} \frac{1}{u}
$$

for all non-negative functions $u$ and $f$.

c) There is a constant $C$ such that

$$
\int_{-\infty}^{\infty}\left[M^{+} f\right]^{p} \frac{1}{\left[M_{X^{\prime}}^{+}\left(u^{1 / p}\right)\right]^{p}} \leq C \int_{-\infty}^{\infty}|f|^{p} \frac{1}{u}
$$

for all non-negative functions $u$, and $f$.

If furthermore $X$ is rearrangement-invariant with fundamental function $\varphi_{X}$,

d) There is a positive constant $c$ such that

$$
\int_{0}^{c} \frac{\varphi_{X}(t)^{p}}{t} \frac{d t}{t}<\infty
$$

Then, $a) \Rightarrow b) \Rightarrow c) \Rightarrow d$ ).

As corollary we have:

Corollary 2.4. Let $1<p<\infty$, and let $X$ be a rearrangement-invariant function space. We assume that there is a positive constant $C$ such that:

$$
\int_{-\infty}^{\infty}\left[M^{+}(f) w\right]^{p} \leq C \int_{-\infty}^{\infty}[|f| v]^{p}
$$

for all $f$, whenever the pair of weights $(w, v)$ satisfies

$$
\left(A_{p, X}^{+}\right) \quad\left(\frac{1}{b-a} \int_{a}^{b} w^{p}\right)^{\frac{1}{p}}\left\|v^{-1}\right\|_{X^{\prime},(b, c)} \leq C
$$

for all $a<b<c: b-a<c-b$. Then, there exists $c>0$ :

$$
\int_{0}^{c} \frac{\varphi_{X}(t)^{p}}{t} \frac{d t}{t}<\infty
$$

Proof. Since the pair $(w, v)=\left(\frac{1}{M_{X^{\prime}}^{+}\left(u^{1 / p}\right)}, \frac{1}{u^{1 / p}}\right)$ satisfies $A_{p, X}^{+}$, this corollary is an immediate consequence of Theorem 2.3, part $c) \Rightarrow d$ ).

The following theorems characterize the spaces such that $M_{X}^{+}$is bounded from $L^{p}$ to $L^{p}$, in the case when $X$ is an Orlicz or Lorentz space. The bilateral case was done by Pérez in [P1] and [P3]. 
A function $B:[0, \infty) \rightarrow[0, \infty)$ is a Young function, if it is continuous, convex, and increasing satisfying $B(0)=0$, and $B(t) \rightarrow \infty$ if $t \rightarrow \infty$. The Luxemburg norm of $f$, given by $B$ is

$$
\|f\|_{B}=\inf \left\{\lambda>0: \int B\left(\frac{|f|}{\lambda}\right) \leq 1\right\}
$$

and so the B-average over $I$ is

$$
\|f\|_{B, I}=\inf \left\{\lambda>0: \frac{1}{|I|} \int_{I} B\left(\frac{|f|}{\lambda}\right) \leq 1\right\} .
$$

We will denote by $\bar{B}$ the complementary function associated to $B$.

Given an Orlicz space $X$ associated to a Young function $B$, we will write $M_{B}^{+}$ instead of $M_{X}^{+}$.

We also recall the definition of the classes $A_{p}^{+}, 1 \leq p<\infty$. The pair $(w, v) \in A_{p}^{+}$ if there exits $C$ such that for every $a<b<c$

$$
\left(\int_{a}^{b} w\right)\left(\int_{b}^{c} v^{1-p^{\prime}}\right)^{p-1} \leq C(c-a)^{p}
$$

for $1<p<\infty$, and

$$
M^{-} w(x) \leq C v(x) .
$$

We say that $w \in A_{\infty}^{+}$, if there exist $\delta>0$ and $C>0$ such that

$$
\frac{|E|}{c-a} \leq C\left(\frac{w(E)}{w(a, b)}\right)^{\delta}
$$

for all $a<b<c$ and every measurable set $E \subset(a, b)$.

Theorem 2.5. Let $(w, v) \in A_{r}^{+}, r \geq 1$, and $B$ a Young function. There exists a constant $C$ such that

$$
w\left\{x: M_{B}^{+} f(x)>t\right\} \leq C \int_{\{x: f(x)>t / 2\}} B^{r}\left(\frac{f}{t}\right) v,
$$

for $f \geq 0$ and $t>0$.

Definition. Let $B$ be a Young function, we say $B \in B_{p}, p>1$, if there exists $c>0$ such that

$$
\int_{c}^{\infty} \frac{B(t)}{t^{p}} \frac{d t}{t}<\infty
$$

Theorem 2.6. Let $(w, v) \in A_{r}^{+}$and $B^{r} \in B_{p}, r \geq 1,1<p<\infty$. Then, there exists a constant $C$ such that

$$
\int\left[M_{B}^{+} f\right]^{p} w \leq C \int|f|^{p} v
$$

for every $f$. 
Corollary 2.7. Let $1<p<\infty$, w a weight, $u$ non-negative, and $B$ a Young function. The following statements are equivalent.

a) $B \in B_{p}$.

b) There exists $C$ such that $\int\left[M_{B}^{+} f\right]^{p} w \leq C \int|f|^{p} M^{-} w$..

c) There exists $C$ such that $\int\left[M^{+} f\right]^{p} \frac{w}{\left[M_{\bar{B}}^{+} u^{1 / p}\right]^{p}} \leq C \int|f|^{p} \frac{M^{-} w}{u}$.

Proof. To see a) $\Longrightarrow \mathrm{b}$ ), we apply Theorem 2.6 to the pair $\left(w, M^{-} w\right) \in A_{1}^{+}$.

For b) $\Longrightarrow \mathrm{c}$ ), we will use that $M^{+}(f g) \leq M_{B}^{+} f M_{\bar{B}}^{+} g$, almost every where, then

$$
\begin{aligned}
\int\left[M^{+} f\right]^{p} \frac{w}{\left[M_{\bar{B}}^{+} u^{1 / p}\right]^{p}} & \leq \int\left[M_{B}^{+} f u^{-1 / p}\right]^{p}\left[M_{\bar{B}}^{+} u^{1 / p}\right]^{p} \frac{w}{\left[M_{\bar{B}}^{+} u^{1 / p}\right]^{p}} \\
& \leq C \int|f|^{p} \frac{M^{-} w}{u} .
\end{aligned}
$$

c) $\Longrightarrow$ a) follows from Theorem 2.3 , in the case of fundamental function $\varphi_{X}(t)=$ $1 / B^{-1}\left(\frac{1}{t}\right)$ and $w=1$.

In this context, Theorem 2.2 has a sort of reciprocal. We will use $A_{p, B, h}^{+}$and $A_{p, B}^{+}$instead of $A_{p, X, h}^{+}$and $A_{p, X}^{+}$respectively.

Corollary 2.8. Let $B$ be a Young function, $1<p<\infty$, and $X$ is the Orlicz space associated to $B$.

a) If $B \in B_{p}$, and $1<p<\infty$, then there exists $C$ such that

$$
\left(\int\left[M_{h}^{+} f\right]^{p} w^{p}\right)^{\frac{1}{p}} \leq C\left(\int|f|^{p} v^{p}\right)^{\frac{1}{p}}
$$

for every pair $(w, v)$ satisfying condition $A_{p, B, h}^{+}$in Theorem 2.2.

b) If $\left(\int\left[M^{+} f\right]^{p} w^{p}\right)^{\frac{1}{p}} \leq C\left(\int|f|^{p} v^{p}\right)^{\frac{1}{p}}$, for every pair $(w, v)$ satisfying condition $A_{p, B}^{+}$in Theorem 2.2, then $B \in B_{p}$.

Proof. First we note, that by Corollary 2.7, $B \in B_{p}$ implies $M_{B}^{+}$is bounded from $L^{p}$ in $L^{p}$ (taking $w=1$ ), and now by Theorem 2.2, we have a).

Observe that b) follows from Corollary 2.4.

Finally as another application let us consider, the Lorentz space $X=L^{s, q}$. A function $f$ belongs to $L^{s, q}, 0<s, q<\infty$, if

$$
\|f\|_{s, q}=\left[q \int_{0}^{\infty}\left(t|\{x \in \mathbb{R}:|f(x)|>t\}|^{1 / s}\right)^{q} \frac{d t}{t}\right]^{1 / q}<\infty
$$

and for $q=\infty$

$$
\|f\|_{s, \infty}=\sup _{0<t<\infty} t|\{x \in \mathbb{R}:|f(x)|>t\}|^{1 / s}<\infty
$$

We will write $M_{s, q}^{+}$instead of $M_{X}^{+}$and $M_{s, q, h}^{+}$instead of $M_{X, h}^{+}$. 
Theorem 2.9. Let $1<s<p<\infty$, and $(w, v) \in A_{l}^{+}, 1 \leq l<\frac{p}{s}$. Then, there exists a constant $C$ such that

$$
\int\left[M_{s, q}^{+} f\right]^{p} w \leq C \int|f|^{p} v
$$

for every $f$.

Corollary 2.10. Let $1<p, s<\infty$ and $1 \leq q<\infty, w$ a weight and $u$ non-negative. The following statements are equivalent.

a) $s<p$

b) There exists $C$ such that

$$
\int\left[M_{s, q}^{+} f\right]^{p} w \leq C \int|f|^{p} M^{-} w
$$

c) There exists $C$ such that

$$
\int\left[M^{+} f\right]^{p} \frac{w}{\left[M_{s^{\prime}, q^{\prime}}^{+} u^{1 / p}\right]^{p}} \leq C \int|f|^{p} \frac{M^{-} w}{u} .
$$

Proof. a) $\Longrightarrow$ b) it follows from Theorem 2.9, observing that the pair $\left(w, M^{-} w\right) \in$ $A_{1}^{+}$.

b) $\Longrightarrow$ c) it is prove in the same way that b) $\Longrightarrow$ c) of Corollary 2.7.

For $\mathrm{c}) \Longrightarrow$ a) we only have to note that the fundamental function in this space is $\varphi(t)=t^{1 / s}$ and that $\int_{0}^{c} \frac{\varphi(t)^{p}}{t} \frac{d t}{t}<\infty$ for some $c>0$ is equivalent to $p>s$.

Corollary 2.11. Let $X=L^{s, q}, 1<p, s<\infty$ and $1 \leq q<\infty$.

a) If $s<p$, then there exists $C$ such that

$$
\left(\int\left[M_{h}^{+} f\right]^{p} w^{p}\right)^{\frac{1}{p}} \leq C\left(\int|f|^{p} v^{p}\right)^{\frac{1}{p}},
$$

for every pair $(w, v)$ satisfying condition $A_{p, X, h}^{+}$in Theorem 2.2.

b) If $\left(\int\left[M^{+} f\right]^{p} w^{p}\right)^{\frac{1}{p}} \leq C\left(\int|f|^{p} v^{p}\right)^{\frac{1}{p}}$, for every pair $(w, v)$ satisfying condition $A_{p, X}^{+}$in Theorem 2.2, then $s<p$.

Proof. It is similar to Corollary 2.8.

In $[\mathrm{AFM}]$, Aimar, Forzani and Martín-Reyes, introduced the one-sided singular integrals. Let us recall some definitions.

We shall say that a function $k$ in $L_{\text {loc }}^{1}(\mathbb{R}-\{0\})$ is a Calderón-Zygmund kernel if the following properties are satisfied:

(a) there exists a finite constant $B_{1}$ such that

$$
\left|\int_{\epsilon<|x|<N} k(x) d x\right| \leq B_{1}
$$

for all $\epsilon$ and all $N$ with $0<\epsilon<N$, and furthermore $\lim _{\epsilon \rightarrow 0^{+}} \int_{\epsilon<|x|<N} k(x) d x$ exists, 
(b) there exists a finite constant $B_{2}$ such that

$$
|k(x)| \leq \frac{B_{2}}{|x|},
$$

for all $x \neq 0$,

(c) there exists a finite constant $B_{3}$ such that

$$
|k(x-y)-k(x)| \leq B_{3}|y||x|^{-2}
$$

for all $x$ and $y$ with $|x|>2|y|>0$.

A one-sided singular integral is

$$
T^{+} f(x)=\lim _{\epsilon \rightarrow 0} \int_{x+\epsilon}^{\infty} k(x-y) f(y) d y,
$$

where $k$ is a Calderón-Zygmund kernel, with support in $\mathbb{R}^{-}$. Examples of such kernels are given in $[\mathrm{AFM}]$. Observe that these are singular integrals. In that paper they show that if $w \in A_{p}^{+}$then $\int\left|T^{+} f\right|^{p} w \leq C \int|f|^{p} w$, for $p>1$, finding in this way, a larger class of weights for which this inequality holds.

For classical singular integrals, Córdoba and Fefferman $[\mathrm{CF}]$ show that,

$$
\int|T f|^{p} w \leq C \int|f|^{p} M_{r} w
$$

where $M_{r} w(x)=M\left(w^{r}\right)(x)^{1 / r}, r>1$. It is not true if we change $M_{r}$ by $M$. Observe also, that $M w \leq C_{1} M^{k} w \leq C_{2} M_{r} w$ for all $k \in \mathbb{N}$ and $r \geq 1$, where $M^{k} w$ is the Hardy-Littlewood maximal operator iterated $k$ times. C. Pérez [P1] asks if (2.12) can be improved by changing $M_{r}$ by some iterated of the maximal function. The answer is yes, moreover he proves that if $T f$ is a singular integral and $w$ is any weight then

$$
\int_{\mathbb{R}}|T f|^{p} w \leq C \int_{\mathbb{R}}|f|^{p} M^{[p]+1} w .
$$

Finally we show that, this result can be improved, if the singular integral is one-sided , replacing $M^{[p]+1} w$ by the smaller operator $\left(M^{-}\right)^{[p]+1} w$ ( the one sided Hardy-Littlewood maximal operator iterated $[p]+1$ times). Actually this result follows from Theorem 2.13.

Theorem 2.13. Let $1<p<\infty$. Let $k$ be a Calderón-Zygmund kernel with support in $\mathbb{R}^{-}$. Let $A$ a Young function such that $B(s)=\overline{A\left(s^{p}\right)} \in B_{p^{\prime}}$ or equivalently there is $c>0$ :

$$
\int_{c}^{\infty}\left[\frac{t}{A(t)}\right]^{p^{\prime}-1} \frac{d t}{t}<\infty .
$$

Then, there exists a constant $C$ such that for each weight $w$

$$
\int_{-\infty}^{\infty}\left|T^{+} f\right|^{p} w \leq C \int_{-\infty}^{\infty}|f|^{p} M_{A}^{-} w .
$$


Proposition 2.15. Let $B(t)=t\left(1+\log ^{+} t\right)^{k}, k \geq 0$, then there exist $c_{1}>0$ and $c_{2}>0$ such that

$$
c_{1} M_{B}^{-} w(x) \leq\left(M^{-}\right)^{k+1} w(x) \leq c_{2} M_{B}^{-} w(x) .
$$

Corollary 2.16. Let $1<p<\infty$, let $k$ be a Calderón-Zygmund kernel with support in $\mathbb{R}^{-}$, then there exists $C$ such that for every weight $w$

$$
\int_{-\infty}^{\infty}\left|T^{+} f\right|^{p} w \leq C \int_{-\infty}^{\infty}|f|^{p}\left(M^{-}\right)^{[p]+1} w
$$

Proof. We only have to observe that the function $A(t)=t\left[1+\log ^{+}(t)\right]^{[p]}$, clearly satisfies

for any $c>0$.

$$
\int_{c}^{\infty}\left[\frac{t}{A(t)}\right]^{p^{\prime}-1} \frac{d t}{t}<\infty
$$

\section{Proof of the theOREMS}

To prove Theorem 2.2 we need to show, that under the assumptions imposed on $h$, we have that $M_{h}^{+} f$ is a continuous function for reasonable $f$.

Lemma 3.1. $M_{h}^{+} f$ is continuous for all $f \geq 0$ continuous and with compact support.

Proof. First we are going to see that $M_{h}^{+} f$, is lower semicontinuous, so we will show that $O_{\lambda}=\left\{x: M_{h}^{+} f(x)>\lambda\right\}$ is open. If $x \in O_{\lambda}$, there exists $c_{x}$ such that $h\left(x, c_{x}\right) \int_{x}^{c_{x}} f>\lambda$. By the continuity of $h$ and continuity of the integral it follows that $M_{h}^{+} f(y)>\lambda$ for $y$ close to $x$.

We will show now that $M_{h}^{+} f$ is upper semicontinuous, by contradiction: suppose there exists $\epsilon>0, x_{0} \in \mathbb{R}$, and $\left\{x_{n}\right\}_{n \geq 1}$, a sequence converging to $x_{0}$ such that

$$
M_{h}^{+} f\left(x_{n}\right)>M_{h}^{+} f\left(x_{0}\right)+\epsilon,
$$

for all $n \geq 1$. For each $n$, there exists $c_{n}>x_{n}$ such that

$$
h\left(x_{n}, c_{n}\right) \int_{x_{n}}^{c_{n}} f>M_{h}^{+} f\left(x_{0}\right)+\epsilon .
$$

Suppose that support of $f$ is contained in $I$, with $|I|<\infty$. As $h$ is non-increasing in the second variable, we can suppose that $c_{n} \in I$ for all $n \geq 1$. So there exist $\left\{c_{n_{j}}\right\}_{j \geq 1}$ such that $c_{n_{j}} \rightarrow c$ when $j \rightarrow \infty$. There are two cases.

1. $c-x_{0}>0$, then continuity of $h$, and $f$ imply

against $\epsilon>0$.

$$
M_{h}^{+} f\left(x_{0}\right) \geq h\left(x_{0}, c\right) \int_{x_{0}}^{c} f>M_{h}^{+} f\left(x_{0}\right)+\epsilon
$$

2. $x_{0}=c$, then

$$
M_{h}^{+} f\left(x_{0}\right)+\epsilon \leq h\left(x_{n_{j}}, c_{n_{j}}\right) \int_{x_{n_{j}}}^{c_{n_{j}}} f=\left(c_{n_{j}}-x_{n_{j}}\right) \frac{h\left(x_{n_{j}}, c_{n_{j}}\right)}{\left(c_{n_{j}}-x_{n_{j}}\right)} \int_{x_{n_{j}}}^{c_{n_{j}}} f
$$

since $\ell f\left(x_{0}\right) \leq M_{h}^{+} f\left(x_{0}\right)$, taking limit we have

$$
\ell f\left(x_{0}\right)+\epsilon \leq \ell f\left(x_{0}\right) .
$$

In the proof of Theorem 2.2 we will use the following lemma. The proof is in Bliendtner and Loeb [BLo]. 
Lemma 3.2. If $\mu$ is a finite Borel measure on $\mathbb{R}$, and if $I$ is an arbitrary collection of non-degenerate intervals, then for each $\delta>0$ there exists a finite subcollection, $I_{\delta}$, of disjoint intervals in I such that

$$
\mu\left(\bigcup_{I \in I} I\right) \leq(2+\delta) \sum_{I_{k} \in I_{\delta}} \mu\left(I_{k}\right)
$$

Proof of Theorem 2.2. Let $f \in L^{p}\left(v^{p}\right)$, non-negative, continuous, and with bounded support. By Lemma 3.1 $M_{h}^{+} f$ is continuous and therefore, $\Omega_{k}=\left\{x: 2^{k}<\right.$ $\left.M_{h}^{+} f(x)<2^{k+1}\right\}$, is open. It easily follows from assumption (2) on $h$ that $M_{h}^{+} f \in$ $L^{s}$ for some $1<s<\infty$. Then, we can assume without loss of generality that the sets $\left\{x: M_{h}^{+} f(x)=2^{k}\right\}$ have measure zero for all integers $k$. Taking into account the definition of $M_{h}^{+} f$, we observe that $\Omega_{k} \subset \bigcup_{x} J_{x, k}$, where $J_{x, k}=\left[x, c_{x}\right)$ satisfies:

$$
2^{k}<h\left(x, c_{x}\right) \int_{x}^{c_{x}} f<2^{k+1}
$$

By the continuity of $h$, and the absolute continuity of the integral, we can define two intervals $I_{x, k}^{-}=\left[x, \delta_{x}\right)$ and $I_{x, k}^{+}=J_{x, k}-I_{x, k}^{-}$such that: $\left|I_{x, k}^{-}\right|<\left|I_{x, k}^{+}\right|, I_{x, k}^{-} \subset \Omega_{k}$, and

$$
2^{k}<h\left(\delta_{x}, c_{x}\right) \int_{\delta_{x}}^{c_{x}} f<2^{k+1} .
$$

By Lemma 3.2, there exists a finite subcollection $\left\{I_{j, k}^{-}\right\}_{j}$ of disjoint intervals such that

$$
w^{p}\left(\Omega_{k}\right) \leq 3 \sum_{j} w^{p}\left(I_{j, k}^{-}\right)
$$

We will use the notation $I_{j, k}^{-}=\left[a_{j, k}, b_{j, k}\right)$, and $I_{j, k}^{+}=\left[b_{j, k}, c_{j, k}\right)$. Now we can make the following estimate:

$$
\begin{aligned}
\int M_{h}^{+} f(x)^{p} w(x)^{p} d x \leq 2^{p} \sum_{k} w^{p}\left(\Omega_{k}\right) 2^{k p} \\
\leq 2^{p} 3 \sum_{k, j} w^{p}\left(I_{j, k}^{-}\right)\left(\frac{\left(c_{j, k}-b_{j, k}\right) h\left(b_{j, k}, c_{j, k}\right)}{\left(c_{j, k}-b_{j, k}\right)} \int_{b_{j, k}}^{c_{j, k}} f v v^{-1}\right)^{p} .
\end{aligned}
$$

¿From the generalized Hölder's inequality for Banach function spaces and the condition $A_{p, X, h}^{+}$, we obtain

$$
\begin{aligned}
(3.3) & \leq 2^{p} 3 \sum_{k, j} w^{p}\left(I_{j, k}^{-}\right)\left(\left(c_{j, k}-b_{j, k}\right) h\left(b_{j, k}, c_{j, k}\right)\|f v\|_{X, I_{j, k}^{+}}\left\|v^{-1}\right\|_{X^{\prime}, I_{j, k}^{+}}\right)^{p} \\
& \leq 2^{p} 3 \sum_{k, j}\left|I_{j, k}^{-}\right|\|f v\|_{X, I_{j, k}^{+}}^{p} \\
& =2^{p} 3 \sum_{k, j} \int_{I_{j, k}^{-}}\|f v\|_{X, I_{j, k}^{+}}^{p}
\end{aligned}
$$


Taking into account hypothesis (2.1) about norms in the Banach function space, the fact that $\left\{I_{j, k}^{-}\right\}_{j, k}$ is a disjoint familly, and the boundedness of $M_{X}^{+}$, we get:

$$
\begin{aligned}
\int M_{h}^{+} f^{p} w^{p} d x & \leq 2^{p} 3 \sum_{k, j} \int_{I_{j, k}^{-}} M_{X}^{+}(f v)^{p} \\
& \leq 2^{p} 3 \int M_{X}^{+}(f v)^{p} \\
& \leq C 2^{p} 3 \int(f v)^{p}
\end{aligned}
$$

Proof of Theorem 2.3. Let us assume a). Since

$$
M_{h}^{+}(f g) \leq M_{X}^{+}(f) M_{X^{\prime}, h}^{+}(g),
$$

we have that

$$
\int_{-\infty}^{\infty}\left[M_{h}^{+} f\right]^{p} \frac{1}{\left[M_{X^{\prime}, h}^{+}\left(u^{1 / p}\right)\right]^{p}} \leq \int_{-\infty}^{\infty}\left[M_{X}^{+}\left(f u^{-1 / p}\right)\right]^{p} \leq C \int_{-\infty}^{\infty}\left|f u^{-1 / p}\right|^{p} .
$$

¿From $b$ ) with $h(x, c)=(c-x)^{-1}$, it follows that $c$ ) holds.

If we consider $c$ ) with $f=u=\chi_{[-1,0]}$, then

$$
\int_{-\infty}^{-2}\left[M^{+} f\right]^{p} \frac{1}{\left[M_{X^{\prime}}^{+}(f)\right]^{p}} \leq C .
$$

Since the inequalities $M_{X^{\prime}}^{+}(f)(y) \leq \varphi_{X^{\prime}}\left(\frac{2}{-y}\right)$ and $M^{+} f(y) \geq \frac{1}{-y}$, hold for every $y \leq-2$, we have that

$$
\frac{1}{2^{p-1}} \int_{0}^{1} \frac{s^{p-1}}{\varphi_{X^{\prime}}(s)^{p}} \frac{d s}{s} \leq C .
$$

Recalling that $\varphi_{X}(s) \varphi_{X^{\prime}}(s) \approx s$, we obtain $\left.d\right)$.

Proof of Theorem 2.5. It will be sufficient to consider bounded nonnegative function $f$ with compact support. Let $t>0$, then $O_{t}=\left\{x: M_{B}^{+} f(x)>t\right\}=\cup_{j}\left(a_{j}, b_{j}\right)$, where the intervals $\left(a_{j}, b_{j}\right)$ are bounded pairwise disjoint. We claim that

$$
1 \leq \frac{1}{b_{j}-x} \int_{x}^{b_{j}} B\left(\frac{f}{t}\right)
$$

for all $x \in\left[a_{j}, b_{j}\right)$. Let $(a, b)$ be one of the intervals $\left(a_{j}, b_{j}\right)$. We will prove (3.4). Let $x \in(a, b)$, then $M_{B}^{+} f(x)>t$. There exists $s>x$ such that $\|f\|_{B,(x, s)}>t$, this implies (using the norm definition) that

$$
\frac{1}{s-x} \int_{x}^{s} B\left(\frac{f}{t}\right)>1
$$

If (3.4) does not hold, then $\|f\|_{B,(x, b)} \leq t$ and since $b \notin O_{t}$, we have that for all $c>b,\|f\|_{B,(b, c)} \leq t$. Then if $s>b$ we have

$$
\int_{x}^{s} B\left(\frac{f}{t}\right)=\int_{x}^{b} B\left(\frac{f}{t}\right)+\int_{b}^{s} B\left(\frac{f}{t}\right) \leq b-x+s-b=s-x,
$$


contradiction. So we have that $s \leq b$. Let $s^{\prime}=\sup \left\{s:\|f\|_{B,(x, s)}>t\right\}$. Notice that $\|f\|_{B,\left(x, s^{\prime}\right)} \geq t$. Suppose $s^{\prime}<b$. As $s^{\prime} \in O_{t}$ then there exists $s^{\prime \prime}$ such that $\|f\|_{B,\left(s^{\prime}, s^{\prime \prime}\right)}>t$. In other words $\frac{1}{s^{\prime \prime}-s^{\prime}} \int_{s^{\prime}}^{s^{\prime \prime}} B\left(\frac{f}{t}\right)>1$. Using that $s^{\prime}$ is the suppremum we have

$$
\int_{s^{\prime}}^{s^{\prime \prime}} B\left(\frac{f}{t}\right)=\int_{x}^{s^{\prime \prime}} B\left(\frac{f}{t}\right)-\int_{x}^{s^{\prime}} B\left(\frac{f}{t}\right) \leq s^{\prime \prime}-x-\left(s^{\prime}-x\right)=s^{\prime \prime}-s^{\prime},
$$

contradiction. So $s^{\prime}=b$ and (3.4) holds.

It will be sufficient to prove that

$$
\int_{a}^{b} w \leq C \int_{a}^{b} B^{r}\left(\frac{f}{t}\right) v
$$

Let $(a, b)=\cup_{k=1}^{\infty}\left(x_{k-1}, x_{k}\right]$, where $\left\{x_{k}\right\}$ is a increasing sequence defined as

$$
x_{0}=a, \text { and } \int_{x_{k-1}}^{x_{k}} B\left(\frac{f}{t}\right)=\int_{x_{k}}^{b} B\left(\frac{f}{t}\right) .
$$

It follows that

$$
\int_{x_{k-1}}^{b} B\left(\frac{f}{t}\right)=4 \int_{x_{k}}^{x_{k+1}} B\left(\frac{f}{t}\right)
$$

Then

$$
1 \leq \frac{1}{b-x_{k-1}} \int_{x_{k-1}}^{b} B\left(\frac{f}{t}\right)=\frac{1}{b-x_{k-1}} 4 \int_{x_{k}}^{x_{k+1}} B\left(\frac{f}{t}\right)
$$

Now, let $r>1$, by Hölder's inequality we get

$$
1 \leq \frac{4^{r}}{\left(b-x_{k-1}\right)^{r}}\left(\int_{x_{k}}^{x_{k+1}} B^{r}\left(\frac{f}{t}\right) v\right)\left(\int_{x_{k}}^{x_{k+1}} v^{-\frac{r^{\prime}}{r}}\right)^{r-1} .
$$

Then by $A_{r}^{+}$

$$
\begin{aligned}
\int_{x_{k-1}}^{x_{k}} w & \leq \frac{4^{r}}{\left(b-x_{k-1}\right)^{r}}\left(\int_{x_{k}}^{x_{k+1}} B^{r}\left(\frac{f}{t}\right) v\right)\left(\int_{x_{k-1}}^{x_{k}} w\right)\left(\int_{x_{k}}^{x_{k+1}} v^{-\frac{1}{r-1}}\right)^{r-1} \\
& \leq C 4^{r} \frac{\left(x_{k+1}-x_{k-1}\right)^{r}}{\left(b-x_{k-1}\right)^{r}} \int_{x_{k}}^{x_{k+1}} B^{r}\left(\frac{f}{t}\right) v \\
& \leq C 4^{r} \int_{x_{k}}^{x_{k+1}} B^{r}\left(\frac{f}{t}\right) v .
\end{aligned}
$$

Summing up in $k$ we get

$$
\int_{a}^{b} w \leq C 4^{r} \int_{x_{1}}^{b} B^{r}\left(\frac{f}{t}\right) v \leq C 4^{r} \int_{a}^{b} B^{r}\left(\frac{f}{t}\right) v .
$$


For $r=1$ we simply observe that from (3.5) and $A_{1}^{+}$we have

$$
\begin{aligned}
\int_{x_{k-1}}^{x_{k}} w & \leq \int_{x_{k-1}}^{x_{k}} w \frac{4}{b-x_{k-1}}\left(\int_{x_{k}}^{x_{k+1}} B\left(\frac{f}{t}\right)\right) \\
& \leq 4 \int_{x_{k}}^{x_{k+1}} B\left(\frac{f(y)}{t}\right) \frac{1}{y-x_{k-1}} \int_{x_{k-1}}^{y} w(x) d x d y \\
& \leq 4 C \int_{x_{k}}^{x_{k+1}} B\left(\frac{f}{t}\right) v
\end{aligned}
$$

and summing up we obtain the same as in the case $r>1$.

Therefore we have

$$
w\left\{x: M_{B}^{+} f(x)>t\right\} \leq C \int_{\left\{x: M_{B}^{+} f(x)>t\right\}} B^{r}\left(\frac{f}{t}\right) v .
$$

To obtain the theorem, we only have to put $f=f_{1}+f_{2}$, where $f_{1}=f \chi_{\{x: f(x)>t / 2\}}$ and $f_{2}=f-f_{1}$. Observe that we can suppose $B(1)=1$, then $M_{B}^{+} f_{2} \leq t / 2$. Now using that $M_{B}^{+}$is bounded from $L^{\infty}$ in $L^{\infty}$ we obtain what we claim.

Proof of Theorem 2.6. Let $f \geq 0$, by Theorem 2.5 we have

$$
\begin{aligned}
\int\left[M_{B}^{+} f\right]^{p} w & =p \int_{0}^{\infty} t^{p} w\left\{x: M_{B}^{+} f(x)>t\right\} \frac{d t}{t} \\
& \leq C p \int_{0}^{\infty} t^{p} \int_{\{x: f>t / 2\}} B^{r}\left(\frac{f(y)}{t}\right) v(y) d y \frac{d t}{t}
\end{aligned}
$$

changing the integration order and using that $B^{r} \in B_{p}$ we get

$$
\int\left[M_{B}^{+} f\right]^{p} w \leq C p \int_{\mathbb{R}}\left(\int_{1 / 2}^{\infty} \frac{B^{r}(s)}{s^{p}} \frac{d s}{s}\right) f^{p}(y) v(y) d y \leq C \int_{\mathbb{R}} f^{p} v .
$$

Proof of Theorem 2.9. Note that $s<r$ and $f \geq 0$, then

$$
\begin{aligned}
\|f\|_{s, 1(x, x+h)} & =\frac{1}{h^{1 / s}}\left\|f \chi_{(x, x+h)}\right\|_{s, 1} \leq C_{s, r} \frac{1}{h^{1 / r}}\left\|f \chi_{(x, x+h)}\right\|_{r, r} \\
& =C_{s, r}\left(\frac{1}{h} \int_{x}^{x+h} f^{r}\right)^{1 / r},
\end{aligned}
$$

then taking supremum we have

$$
M_{s, 1}^{+} f(x) \leq C_{s, r} M_{r}^{+} f(x)
$$

Let us consider $r$ such that $s<r<p$, and $l<\frac{p}{r}$. Then $M^{+}\left(|f|^{r}\right)$ is strong type from $L^{p / r}(v)$ in $L^{p / r}(w)$ (see [S] or [MOT]). So we have

$$
\int\left[M_{s, t}^{+} f\right]^{p} w \leq \int\left[M_{s, 1}^{+} f\right]^{p} w \leq C \int\left[M_{r}^{+} f\right]^{p} w \leq C \int\left(f^{r}\right)^{p / r} v
$$


Proof of Theorem 2.13. By duality (2.14) is equivalent to

$$
\int_{-\infty}^{\infty}\left|T^{-} f\right|^{p^{\prime}}\left[M_{A}^{-} w\right]^{1-p^{\prime}} \leq C \int_{-\infty}^{\infty}|f|^{p^{\prime}} w^{1-p^{\prime}}
$$

Since $\left[M_{A}^{-} w\right]^{1-p^{\prime}}=\left[\left(M_{A}^{-} w\right)^{\frac{p^{\prime}-1}{r-1}}\right]^{1-r}$ for all $r>p^{\prime}$, we have that $\left[M_{A}^{-} w\right]^{1-p^{\prime}} \in$ $\bigcap_{r>p^{\prime}} A_{r}^{-} \subseteq A_{\infty}^{-}$. Then, by Theorem 2.1 in $[\mathrm{AFM}]$, we get

$$
\int_{-\infty}^{\infty}\left|T^{-} f\right|^{p^{\prime}}\left[M_{A}^{-} w\right]^{1-p^{\prime}} \leq C \int_{-\infty}^{\infty}\left|M^{-} f\right|^{p^{\prime}}\left[M_{A}^{-} w\right]^{1-p^{\prime}}
$$

If $B(t)=\overline{A\left(t^{p}\right)}$, then $M_{A}^{-}\left(f^{p}\right)=\left[M_{\bar{B}}^{-} f\right]^{p}$, so for $f=w^{p^{\prime}-1}$ we have $\left[M_{\bar{B}}^{-} w^{\frac{p^{\prime}-1}{p^{\prime}}}\right]^{p^{\prime}}=$ $\left[M_{\bar{B}}^{-} w^{\frac{1}{p}}\right]^{p^{\prime}}=\left[M_{A}^{-} w\right]^{p^{\prime}-1}$. Observe that the pair $\left(\left[M_{\bar{B}}^{-} w^{\frac{1}{p}}\right]^{-1}, w^{-\frac{1}{p}}\right) \in A_{p^{\prime}, B}^{-}$and $B \in B_{p^{\prime}}$. We can apply Theorem 2.2 and obtain

$$
\begin{aligned}
\int_{-\infty}^{\infty}\left|T^{-} f\right|^{p^{\prime}}\left[M_{A}^{-} w\right]^{1-p^{\prime}} & \leq C \int_{-\infty}^{\infty}\left|M^{-} f\right|^{p^{\prime}}\left[M_{\bar{B}}^{-} w^{\frac{1}{p}}\right]^{-p^{\prime}} \\
& =C \int_{-\infty}^{\infty}\left|M^{-} f\right|^{p^{\prime}}\left[M_{\bar{B}}^{-}\left(w^{\frac{p^{\prime}}{p}}\right)^{\frac{1}{p^{\prime}}}\right]^{-p^{\prime}} \leq C \int_{-\infty}^{\infty}|f|^{p^{\prime}} w^{-\frac{p^{\prime}}{p}}
\end{aligned}
$$

This concludes the proof of the theorem.

Proof of Proposition 2.15. Let us consider $A(t)=t\left(\log ^{+} t\right)^{k}$. We will prove $M_{A}^{-} w \leq$ $C\left(M^{-}\right)^{k+1} w$. Let $O_{\lambda}=\left\{x: M^{-} w(x)>\lambda\right\}$. It is well known that

$$
\frac{1}{\lambda} \int_{O_{\lambda}} w=\left|O_{\lambda}\right|
$$

Integrating both sides of (3.6) we get

$$
\int_{1}^{\infty} \frac{1}{\lambda} \int_{O_{\lambda}} w(x) d x d \lambda=\int_{1}^{\infty} \int_{O_{\lambda}} 1 d x d \lambda \leq \int_{\left\{x: M^{-} w(x)>1\right\}} M^{-} w(x) d x .
$$

For the left hand side we have

$$
\begin{aligned}
\int_{1}^{\infty} \frac{1}{\lambda} \int_{O_{\lambda}} w(x) d x d \lambda & \geq \int_{1}^{\infty} \frac{1}{\lambda} \int_{\{x: w(x)>\lambda\}} w(x) d x d \lambda \\
& =\int_{\{x: w(x)>1\}} w(x) \int_{1}^{w(x)} \frac{1}{\lambda} d \lambda d x \\
& =\int_{\{x: w(x)>1\}} w(x) \log w(x) d x
\end{aligned}
$$

So we get

$$
\int_{\{x: w(x)>1\}} w(x) \log ^{+} w(x) d x \leq \int_{\left\{x: M^{-} w(x)>1\right\}} M^{-} w(x) d x .
$$


We observe that from (3.7) it follows

$$
\int_{\{x: w(x)>\lambda\}} \frac{w(x)}{\lambda} \log +\frac{w(x)}{\lambda} d x \leq \int_{O_{\lambda}} M^{-}\left(\frac{w(x)}{\lambda}\right) d x,
$$

for all $\lambda>0$. Integrating (3.8) several times from $\lambda=1$ to $\infty$ we get

$$
\begin{aligned}
& \int_{\{x: w(x)>\lambda\}} \frac{w(x)}{\lambda} {\left[\log +\frac{w(x)}{\lambda}\right]^{k} d x } \\
& \leq k \int_{O_{\lambda}} M^{-}\left(\frac{w(x)}{\lambda}\right)\left[\log ^{+} M^{-}\left(\frac{w(x)}{\lambda}\right)\right]^{k-1} d x,
\end{aligned}
$$

for all $k \in \mathbb{N}$ and $\lambda>0$. Iterating (3.9) it follows

$$
\int_{\{x: w(x)>\lambda\}} \frac{w(x)}{\lambda}\left[\log ^{+} \frac{w(x)}{\lambda}\right]^{k} d x \leq k ! \int_{\left\{x:\left(M^{-}\right)^{k} w(x)>\lambda\right\}}\left(M^{-}\right)^{k}\left(\frac{w(x)}{\lambda}\right) d x
$$

for all $\lambda>0$.

Let us consider now, $I=(a, b), \lambda_{0}=k !\left(M^{-}\right)^{k+1}\left(w \chi_{I}\right)(b)$, and $w \chi_{I}$. Observe that if $x<a,\left(M^{-}\right)^{k}\left(w \chi_{I}\right)(x)=0$, and if $x>b,\left(M^{-}\right)^{k}\left(w \chi_{I}\right)(x) \leq$ $\left(M^{-}\right)^{k}\left(w \chi_{I}\right)(b) \leq \lambda_{0}$. Therefore $\left\{x:\left(M^{-}\right)^{k}\left(w \chi_{I}\right)(x)>\lambda_{0}\right\} \subset I$. It follows from (3.10), applied to $w \chi_{I}$ that

$$
\begin{aligned}
\frac{1}{|I|} \int_{I} A\left(\frac{w}{\lambda_{0}}\right) & =\frac{1}{|I|} \int_{I}\left(\frac{w}{\lambda_{0}}\right)\left[\log ^{+}\left(\frac{w}{\lambda_{0}}\right)\right]^{k} \\
& =\frac{1}{|I|} \int_{\left\{x \in I: w \chi_{I}>\lambda_{0}\right\}}\left(\frac{w}{\lambda_{0}}\right)\left[\log ^{+}\left(\frac{w}{\lambda_{0}}\right)\right]^{k} \\
& \leq \frac{k !}{|I|} \int_{\left\{x:\left(M^{-}\right)^{k}\left(w \chi_{I}\right)(x)>\lambda_{0}\right\}}\left(M^{-}\right)^{k}\left(\frac{w \chi_{I}}{\lambda_{0}}\right) \leq \frac{k !}{|I| \lambda_{0}} \int_{I}\left(M^{-}\right)^{k}\left(w \chi_{I}\right) \\
& \leq \frac{k !}{\lambda_{0}}\left(M^{-}\right)^{k+1}\left(w \chi_{I}\right)(b)=1 .
\end{aligned}
$$

Recalling that

$$
\|w\|_{A, I}=\inf \left\{\lambda>0: \frac{1}{|I|} \int_{I} A\left(\frac{w}{\lambda}\right) \leq 1\right\}
$$

we have $\|w\|_{A, I} \leq \lambda_{0}=k !\left(M^{-}\right)^{k+1}\left(w \chi_{I}\right)(b)$, then

$$
M_{A}^{-} w(b) \leq k !\left(M^{-}\right)^{k+1} w(b) .
$$

Also observe that if $C$ and $D$ are Young functions then $M_{C+D}^{-} w(x) \leq M_{C}^{-} w(x)+$ $M_{D}^{-} w(x)$. So we have that

$$
M_{B}^{-} w(x) \leq C k !\left(M^{-}\right)^{k+1} w(x) .
$$

To prove the other inequality we will do it for $k=1$, since it is not very difficult to see the general case. Let $I=(a, b)$ and $w=w_{1}+w_{2}$ where $w_{1}=w \chi_{(2 a-b, b)}=w \chi_{2 I}$, and $w_{2}=w-w_{1}$. We have that

$$
\frac{1}{|I|} \int_{I} M^{-} w \leq \frac{1}{|I|} \int_{I} M^{-} w_{1}+\frac{1}{|I|} \int_{I} M^{-} w_{2}
$$


Observe that for all $x \in I, M^{-} w_{2}(x) \leq M^{-} w(b)$, so

$$
\frac{1}{|I|} \int_{I} M^{-} w_{2} \leq M^{-} w(b) \leq M_{B}^{-} w(b)
$$

Now we have to see that

$$
\frac{1}{|2 I|} \int_{2 I} M^{-} w_{1} \leq C\|w\|_{B, 2 I}
$$

We can suppose $\|w\|_{B, 2 I}=1$, and this implies

$$
\frac{1}{|2 I|} \int_{2 I} B(w)=\frac{1}{|2 I|} \int_{2 I} w\left(1+\log ^{+}(w)\right) \leq 1 .
$$

Then, it is enough to prove

$$
\frac{1}{|2 I|} \int_{2 I} M^{-} w_{1} \leq C\left(1+\frac{1}{|2 I|} \int_{2 I} w \log ^{+}(w)\right) .
$$

This last inequality is valid for $M w$ instead of $M^{-} w$ (see [GR] p.147). Using that $M^{-} w \leq M w$, we have what we stated. Finally

$$
\frac{1}{|I|} \int_{I} M^{-} w \leq C M_{B}^{-} w(b)+C\|w\|_{B, 2 I},
$$

taking sup we have

$$
\left(M^{-}\right)^{2} w(x) \leq C M_{B}^{-} w(x)
$$

\section{REFERENCES}

[AnS] K.F. Andersen and E. Sawyer, Weighted norm inequalities for Riemann-Liouville and Weyl fractional integral operators, Trans. Amer. Math. Soc. 308 (1988), 547-557.

[AFM] H. Aimar, L. Forzani, F.J. Martín-Reyes, On weighted inequalities for one-sided singular integrals, Proc. Amer. Math. Soc. 125 (1997), 2057-2064.

[BLo] J. Bliendtner and P. Loeb, A reduction technique for limit theorems in analysis and probability theory, Ark.Mat 30 (1992), 25-43.

[CF] A. Córdoba and C. Fefferman, A weighted norm inequality for singular integrals, Studia Math. 57 (1976), 97-101.

[GR] J. García Cuerva and J.L. Rubio de Francia, Weighted norm inequalities and related topics, North-Holland Math.Studies, vol. 116, North-Holland, Amsterdam, 1985.

[LT] M. Lorente and A. de la Torre., Weighted inequalities for some one-sided operators, Proc. Amer. Math. Soc. 124 (3) (1996), 839-848.

[MOT] F.J. Martín-Reyes, P. Ortega, A. de la Torre, Weighted Inequalities for One-Sided Maximal Functions, Trans. Amer. Math. Soc. 319-2 (1990), 517-534.

[MT] F. Martín-Reyes and A. de la Torre., Two weight inequalities for fractional one-sided maximal operators, Proc. Amer. Math. Soc. 117 (2) (1993), 483-489.

[P1] C. Pérez, On sufficient conditions for the boundedness of the Hardy-Littlewood maximal operator between weighted $L^{p}$-spaces with different weights, Proc. London Math. Soc. 71 (3) (1995), 135-157.

[P2] Weighted norm inequalities for singular integral operators, J.London Math. Soc. 49 (1994), 296-308.

[P3] _ Two weighted inequalities for potencial and fractional type maximal operators, Indiana Univ. Math. 43 (1994), 1-28. 
[S] E. Sawyer, A characterization of a two-weighted norm inequality for maximal operators, Studia Math. 75 (1982), 1-11.

Fa.MAF Universidad Nacional de Córdoba. 5000 Córdoba, Argentina.

E-mail address: sriveros@mate.uncor.edu

Dpto. de Matemática, Facultad de Ciencias Exactas y Naturales, Universidad de Buenos Aires. 1428 Buenos Aires, Argentina.

E-mail address: Iderosa@mate.dm.uba.ar

Análisis Matemático. Facultad de Ciencias. Universidad de Málaga. 29071 MáLAGA, SPAIN.

E-mail address: torre@anamat.cie.uma.es 